\title{
Paweł Moskal \\ Few-Body Aspects of the Near Threshold Pseudoscalar Meson Production
}

Received: 29 January 2014 / Accepted: 17 February 2014 / Published online: 9 March 2014

(C) The Author(s) 2014. This article is published with open access at Springerlink.com

\begin{abstract}
During the last decade large samples of data have been collected on the production of the groundstate pseudoscalar mesons in collisions of proton or deuteron beam with hydrogen or deuterium target. These measurements have been performed in the vicinity of the kinematical threshold for meson production where only a few partial waves in both initial and final state are expected to contribute to the production process. This simplifies significantly the interpretation of the data, yet still appears to be challenging due to the three or four particle final state systems with a complex hadronic potential. We review experiments and phenomenology of the near threshold production of the ground-state mesons in the few-body final states as for example: nucleus-meson and nucleon-nucleon-meson, and report on the status of the search of the mesic-nuclei (a meson-nucleus bound states). Experimental advantages of measurements close to the kinematical threshold are discussed, and general features of the production mechanism of the $\eta$ and $\eta^{\prime}$ mesons in the nucleon-nucleon collisions are presented emphasising results of measurements of spin and isospin dependence of the production cross sections.
\end{abstract}

\section{Introduction}

The organisers encouraged the author to summarize the threshold meson production studies conducted so far. However, due to the limitations in the number of pages this contribution cannot be treated as a summary. It is rather a very brief description of some of the studies on the meson production at threshold reflecting the interest of the author. We will concentrate on the $\eta$ and $\eta^{\prime}$ mesons and the report will be restricted to the hadronic processes whereas for the photo-production the interested reader is referred e.g. to [1].

Studies of the production of mesons in the collisions of hadrons are conducted in order to learn about the structure of these mesons, their production mechanisms and about their hadronic interactions with nucleons [2-5]. Measurements and interpretation of results of hadronic processes in the energy regime of one $\mathrm{GeV}$ is challenging since in low energy Quantum Chromodynamics the processes involving strong interactions cannot be treated perturbatively and the analysis must rely on effective field theories and models. The $\eta$ and $\eta^{\prime}$ mesons are short lived and it is unfeasible to accomplish out of them a beam or target, and thus their interaction with other hadrons cannot be investigated in the standard way via scattering experiments. However, production of these mesons close to the kinematical threshold with low relative velocities to nucleons gives a chance to study their interaction with nucleons which may manifest itself as structures in a meson-nucleon invariant mass

P. Moskal (ه)

Institute of Physics, Jagiellonian University, Cracow, Poland

E-mail: p.moskal@uj.edu.pl

Tel.: +48-12-6635558

Fax: +48-12-6637086

P. Moskal

IKP, Forschungszentrum Juelich, Jülich, Germany 
distributions and as enhancements in the cross section excitation functions with respect to predictions based on the assumption that the kinematically available phase space is homogeneously populated.

Interpretation of measurements near the threshold is simplified due to the strongly suppressed contribution form the higher partial waves, due to the low (tending to zero at threshold) relative momentum between the outgoing particles and because of small distances at which the creation of the meson accurs. But nevertheless it remains challenging to discern effects of the meson-nucleon interaction from the overwhelming nucleonnucleon interaction. This is why fifty years after the discovery of the $\eta$ and $\eta^{\prime}$ mesons [6-8] their hadronic interaction with nucleons is still not well known. In the case of the $\eta^{\prime}$ meson its threshold production e.g. via the $p p \rightarrow p p \eta^{\prime}$ reaction occurs at distances of the colliding nucleons in the order of $0.2 \mathrm{fm}$. Therefore, gluonic degrees of freedom may play a role in the production processes [9], implying that for the description of the meson production at threshold both hadronic and quark-gluon degrees of freedom should be considered.

Meson production at threshold is dominated by an S-wave in the final state but at the same time threshold "filters" a single partial wave in the initial state. For example, protons collide predominantly in the ${ }^{3} P_{0}$ state [2] in the case of the pseudoscalar meson production in proton-proton interactions.

Close-to-threshold the production cross section of pseudoscalar mesons is reduced by the initial state interaction (ISI) of the colliding nucleons by a factor of about $4[10,11]$ and it is enhanced by the final state interaction (FSI) by more than an order of magnitude [2]. The reduction of the cross section due to the ISI strongly depends on the meson mass and therefore as it was introduced in reference [12], the strength of the production dynamics is at best expressed as the total cross section normalized to the ISI factor. A natural variable for comparing the production dynamics for different mesons, making it approximately independent of the meson mass, is the volume of the available phase space [12]. Such comparison was made for the $\pi^{0}, \eta$ and $\eta^{\prime}$ mesons and it was shown that the dynamics for the $\eta$ meson production is about six time stronger than for the $\pi^{0}$ meson, which again is by further factor of six stronger than that of the $\eta^{\prime}$ [12].

A suppressed contribution of higher than $l=0$ partial waves is of a great advantage for the measurements at threshold. However, a precise quantitative determination of their contributions is important especially for the studies of the meson nucleon interaction. This is because influence of the meson-nucleon interactions on the cross sections distributions is small and may be easly burdened by the effects caused by the higher partial waves. E.g. enhancements in the meson-nucleon invariant mass distribution due to the meson-nucleon interaction may be mixed with effects due to the p-wave nucleon-nucleon production $[13,14]$. Experimental determination of contributions from various partial waves is difficult and requires measurements with polarised beams and targets. So far such measurements were performed only for the $\pi^{0}$ meson production $[15,16]$. In case of the $\eta$ mesons only beam analysing power $A_{y}$ for the $p p \rightarrow p p \eta$ reaction was studied with a poor precision [17-20] where the best result of $A_{y}$ determined for four angular bins with uncertainty of \pm 0.1 is based on about 2000 reconstructed events [17]. In the near future, the precision of the determination of angular dependence of the analysing power for the $p p \rightarrow p p \eta$ reaction will be improved up to \pm 0.01 based on a new high statistics data sample (about $10^{6}$ reconstructed $p p \rightarrow p p \eta$ events) collected using the large acceptance and azimuthally symmetric WASA detector and a polarized proton beam of the Cooler Synchrotron COSY [21,22]. However, the author is not aware of any plans of measurements of the spin observables for the $\eta^{\prime}$ meson production in the collissions of nucleons.

\section{Experimental Advantages of the Threshold Meson Production}

In the case of the fixed target experiments the fast movement of the center-of-mass system along the beam line cause, for the close to threshold reactions, that all ejectiles are confined in a narrow cone and can be efficiently registered with the detectors of relatively small sizes. The emission of all ejectiles under small angles enables to use dipole magnets as charged particles analyzers for momentum determination with high precision in the whole available phase space volume. A description of the typical zero-degree facilities dedicated to the studies of the threshold meson production can be found e.g. in [23-25]. In addition, in the case of the missing mass techique used for the identification of the produced mesons, a measurement at threshold improves mass resolutions because at threshold the partial derivative of the missing mass with respect to the momentum of outgoing ejectile tends to zero [26]. Moreover, close to threshold the signal-to-background ratio increases due to the more rapid reduction of the phase space for multimeson production than for the single meson [26].

As examples of successful measurements benefiting from the threshold kinematics it is worth to mention: (i) Determination of the natural width of $\eta^{\prime}$ meson directly from its mass distribution for the first time with the resolution comparable to its width $(\sim 200 \mathrm{KeV})[26]$ and (ii) determination of the value of the mass of the $\eta$ 
meson [28] with high precision based on the close-to-threshold measurement of the $d p \rightarrow{ }^{3} \mathrm{He \eta}$ reation where the $\eta$ meson was identified via missing mass distribution and the individual beam momenta were fixed with a relative precision of $d p / p \sim 3 \cdot 10^{-5}$ using a polarized deuteron beam of COSY and inducing an artificial depolarizing spin resonance [29]. It is worth to stress that the precision of the determination of the width of $\eta^{\prime}$ meson by means of the COSY-11 detector [26] was equal to the accuracy which Particle Data Group had previously achieved by combining 51 experiments which determined properties of the eta-prime meson only indirectly connected with its width [27].

The above discussed advantages of the meson production at threshold are, however, valid only for studies of mesons with a narrow spectral functions. In the the case of broad resonances (as e.g. $f_{0}$ or $a_{0}$ ) the notion close-to-threshold becomes non-trivial. In this case a phase space volume varies significantly within the mass range of the meson and the determination of cross section requires a scanning of the excess energies in the range of the spectral function of the studied meson. A formal definition of the close-to-threshold total cross section for broad resonances was first introduced in reference [30], where it was applied to the interpretation of the threshold production of the $f_{0}$ meson in the collisions of protons [30].

\section{Threshold Production of the $\eta$-Nucelon-Nucleon and $\eta^{\prime}$-Nucleon-Nucleon Systems}

The near threshold production of the $\eta$ meson was intensively studied in nucleon-nucleon collisions for which a total [31-35] and differential [13,36-38] cross sections have been established for the $p p \rightarrow p p \eta$ reaction as well as for the $p n \rightarrow p n \eta$ process [39-41]. In case of the $p p \rightarrow p p \eta$ reaction an angular dependence of the analysing power $A_{y}$ has also been established [17-20]. It is important to stress that results from different laboratories (CELSIUS, COSY, SATURNE) are consistent within the estimated systematical uncertainties which are in the order of $10 \%$.

The proton-neutron reaction were realized via quasi-free proton-neutron collisions using a deuteron as a source of neutrons. In the data analysis it was assumed that a spectator proton leaves the deuteron undisturbed and that it is on mass shell already at the collision moment and that the matrix element for the production of the $\eta$ meson by the beam proton off the neutron bound in the deuteron is identical to that for the free $p n \rightarrow p n \eta$ reaction [2,3]. These assumptions are well supported by theoretical considerations [42] and were confirmed by many experiments $[39,41,43-46]$ which have proven that spectator model is valid at least for Fermi momentum up to $150 \mathrm{MeV} / \mathrm{c}$ [44].

The strong dependence of the $\eta$ meson production on the isospin of the colliding nucleons was observed. Total cross sections for the quasi-free $p n \rightarrow p n \eta$ exceed corresponding cross sections for the $p p \rightarrow p p \eta$ reaction by a factor of about three at thereshold [41] and by factor of six at higher excess energies [40]. Combining information of the strong isospin dependence and the isotropic angular distributions of the $\eta$ meson emission angle in the center-of-mass frame, it was established that the $\eta$ meson is predominantly created via excitation of one of the nucleons to the $N^{*}$ (1535) resonance via exchange of the isovector meson [5] and the angular dependence of the analysing power slightly indicated that the process proceed via exchange of the $\pi$ meson [17].

In the case of the $\eta^{\prime}$ meson production in the collisions of nucleons almost 40 times smaller cross sections were observed [34,47-51] than in the case of the $\eta$ meson, which indicates that in contrast to the $\eta$ meson the $\eta^{\prime}$ is produced non-resonantly. Based on the comparison of the shape of the excitation functions for the $p p \rightarrow p p \eta$ and $p p \rightarrow p p \eta^{\prime}$ reactions it was concluded that the $p-\eta$ interaction is much stronger than the $p-\eta^{\prime}$ [12]. At first, the small values of the cross sections for the $p p \rightarrow p p \eta^{\prime}$ reaction [47] were even interpreted as an indication of the $p-\eta^{\prime}$ repulsive interaction [52]. This hypothesis was however excluded later by the more precise data [48].

Up to now, as regards the interaction of pseudoscalar mesons with nucleons the only well known is this of $\pi^{0}$ for which a real part of the scattering legth is equal to $0.1294 \pm 0.0009 \mathrm{fm}$ [53]. The values of the real part of the $p-\eta$ scattering length are known much less precisely and varies from 0.20 to $1.05 \mathrm{fm}$ depending on the analysis method [54-57]. Differences in the value of $\eta N$ scattering lengths obtained in different analyses are at least to some extent explained by the recent observation that the flavour singlet component induces greater binding than the flavour-octet one [58,59]. Therefore, the $\eta-\eta^{\prime}$ mixing, which is neglected in many of the former analyses, increase the $\eta$-nucleon scattering length relative to the pure octet $\eta$ by a factor of about 2 [59]. For the sake of completness, it is important to stress that based on the close to threshold cross sections measured for the $p p \rightarrow p p K^{+} K^{-}$reaction [60-68] the scattering length and effective range of the interaction between strange pseudoscalar mesons $K^{+} K^{-}$were recently estimated [65,69]. The resultant values are consistent with zero with rather large uncertaintes. 
One of the interesting, and still not fully understood observations from the threshold production of $\eta$ and $\eta^{\prime}$ mesons, are large enhancements in invariant masses of two-particle subsystems seen both in the $p p \eta[13,36,37]$ and $p p \eta^{\prime}$ [14] systems. Since the enhancement is similar in $\eta$ and $\eta^{\prime}$ case [14], and the strength of proton- $\eta$ and proton- $\eta^{\prime}$ interaction seems to be different [12], one can conclude that the observed enhancement is not caused by a proton-meson interaction, especially that calculations assuming a significant contribution of Pwave in the final state [70], and models including energy dependence of the production amplitude [71,72], reproduce the data within error bars. Therefore, the determination of the spin observables and extraction of the contribution from the higher partial waves is mandatory for the understanding of the observed enhancements in the discussed invariant mass spectra.

It is also instructive to determine the isospin dependece of the production cross sections which enables to disentangle contributions from various meson exchanges in the reaction mechanisms and at the same time enables to learn about the structure of the produced mesons. In this context especially interesting is the gluonic content of the $\eta^{\prime}$ meson which is discussed comprehensively in articles $[9,58,73,74]$, where it is argued that a comparison of the close-to-threshold total cross sections for the $\eta^{\prime}$ meson production in both the $p p \rightarrow p p \eta^{\prime}$ and $p n \rightarrow p n \eta^{\prime}$ reactions should provide insight into the flavour-singlet (perhaps also into gluonic) content of the $\eta^{\prime}$ meson and the relevance of quark-gluon or hadronic degrees of freedom in the creation process. The production through the colour-singlet object as suggested in reference [9] is isospin independent and should lead to the same production yield of the $\eta^{\prime}$ meson in the $p n \rightarrow$ pn gluons $\rightarrow p n \eta^{\prime}$ and $p p \rightarrow p p$ gluons $\rightarrow p p \eta^{\prime}$ reactions after correcting for the final and initial state interaction between the nucleons [3]. In the case of the $\eta$ meson production in collisions of nucleons the creation from isospin $\mathrm{I}=0$ exceeds the production with $\mathrm{I}=1$ by a factor of about 12, indicating production through the exchange of isovector objects. However in case of the $\eta^{\prime}$ meson so far only an upper limits of cross sections for $\mathrm{I}=0$ have been established [75]. The result indicates weaker isospin dependence for the $\eta^{\prime}$ meson production with respect to the $\eta$ meson, and disfavours the dominance of the $N^{*}(1535)$ resonance in the production process of the $\eta^{\prime}$ meson. It is also worth mentioning that similar effects to the gluonic production were predicted within a meson exchange model [76]. However the so far achieved accuracy is not suffcient to conclude about a gluonic content in the $\eta^{\prime}$ meson.

\section{Threshold Production of the $\eta-{ }^{3} \mathrm{He}$ and $\eta-{ }^{4} \mathrm{He}$ Systems and the Search for the Eta-Mesic Helium}

The production of the $\eta$ meson has been also studied intensively in the proton-deuteron and deutron-deutron reactions e.g.: $p d \rightarrow{ }^{3} \mathrm{He \eta}$ [77-83], $d d \rightarrow{ }^{4} \mathrm{He \eta}$ [84-87], and $p d \rightarrow p d \eta$ [88-90]. Similarly as in the case of the nucleon-nucleon-meson final state a large enhancement of the cross sections over the predictions based on the assumption of the homogeneously populated phase space was observed at threshold for all listed reactions. The steepest rise of the total cross section is seen for the $p d \rightarrow{ }^{3} \mathrm{He \eta}$ reaction. It grows from zero up the the value of about $400 \mathrm{nb}$ over the range of $1 \mathrm{MeV}$ of excess energy and next keeps almost constant in the excess energy range of about $10 \mathrm{MeV}$. This enhancement may be assigned to the ${ }^{3} \mathrm{He}-\eta$ interaction, because it is also observed in the photo-production reactions [91-93], thus it is independent on the initial channel, and because the s-wave production amplitude is fairly energy independent $[78,94,95]$. Moreover, the asymmetry in the angular distribution of the $\eta$ meson emission $[82,83]$ indicates strong changes of the phase of the s-wave production amplitude with energy, as expected from the occurrence of the bound or virtual $\eta^{3} \mathrm{He}$ state [96]. Again similar behaviour is observed in photo-production reactions where evolution of the angular dependence of $\gamma^{3} \mathrm{He} \rightarrow \eta^{3} \mathrm{He}$ [93] as a function of energy, indicates changing of s-wave amplitude associated with the $\eta^{3} \mathrm{He}$ pole [94].

Existence of a bound state of the $\eta$ meson and nucleus (referred to as a mesic nucleus) was predicted 28 years ago [97]. Initially the $\eta$-mesic nuclei were considered to exists for $A \geq 12$ only [97] due to the relatively small value of the $\eta \mathrm{N}$ scattering length estimated in eighties [98]. A decade later, a large values of the $\eta$-nucleon scattering length $(1 \mathrm{fm})$ were extracted in some analysis reported in [99] which do not exclude the formation of bound $\eta$-nucleus states for such light nuclei as helium [100,101] or even for deuteron [102]. However, so far none of the experiments have confirmed univocally the existence of such a state neither in reactions induced by pions [103], protons [104], deuterons [105-107] or photons [93,108]. In the searches for the direct signal from the $\eta$-mesic helium the established so far upper limits amount to about $270 \mathrm{nb}$ for the $d p \rightarrow\left({ }^{3} \mathrm{He} \eta\right)_{\text {bound }} \rightarrow p p p \pi^{-}$reaction [105], about $70 \mathrm{nb}$ for the $d p \rightarrow\left({ }^{3} \mathrm{He} \eta\right)_{\text {bound }} \rightarrow{ }^{3} \mathrm{He} \pi^{0}$ reaction [105], and about $25 \mathrm{nb}$ for the $d d \rightarrow\left({ }^{4} \mathrm{He} \eta\right)_{\text {bound }} \rightarrow{ }^{3} \mathrm{He} p \pi^{-}$reaction [106]. The determined upper limits are close to the newly predicted values of total cross sections for the dd and pd reaction at the $\eta$-mesic pole [94,109], which amounts to about $80 \mathrm{nb}$ for the $p d \rightarrow\left({ }^{3} \mathrm{He}-\eta\right)_{\text {bound }} \rightarrow X p \pi^{-}$[94] and is in the 
range from 4.5 [109] to $30 \mathrm{nb}$ for the $d d \rightarrow(4 \mathrm{He}-\text { eta })_{\text {bound }} \rightarrow X p \pi^{-}$reaction [94]. Although so far not successful, the experimental [1,110-112] and theoretical $[4,58,59,109,113-117]$ investigations of the $\eta$-mesic nucleus are being continued. The observation of such a bound state and determination of its properties would be very valuable for the determination of the $\eta$-nucleon interaction, the $\mathrm{N}^{*}(1535)$ properties in nuclear matter [117,118], the properties of the $\eta$ meson in the nuclear medium $[99,114,119]$, and in general the studies of the chiral and axial U(1) symmetry breaking in low energy QCD $[59,73,117]$. The properties of $\eta$ and $\eta^{\prime}$ mesic nuclei are strongly sensitive to the contribution of the flavour-singlet component of these mesons. Therefore, the $\eta^{\prime}$-mesic nucleus is very interesting in this context too.

The quark condesate is modified in nucleus which changes the properties of hadrons in nuclear medium. The binding energies of $\eta$ and $\eta^{\prime}$ in medium are sensitive to the non-perturbative glue associated with the axial U(1) dynamics [59,73], and as suggested in [120] "due to the UA(1) anomaly effect, a relatively large mass reduction of $\eta^{\prime}$ meson is expected at nuclear saturation density, which may indicate the existence of the $\eta^{\prime}$-mesic nucleus". However, so far most of the experimental studies have been concentrated on the search for the $\eta$-mesic nuclei because the $\eta$-nucleon interaction seems to be much stronger than the $\eta^{\prime}$-nucleon or $\pi$-nucleon [12]. Yet, recently there are vigourous theoretical [59,117,121-123] and experimental [124-126] investigations of feasibility of the observation of the $\eta^{\prime}$-mesic nuclei started by the predictions published in [127]. Based on the cross sections from the $p p \rightarrow p p \eta^{\prime}$ reactions a scattering length of the $p-\eta^{\prime}$ potential seems to be small [12], on the other hand recent photoproduction measurements of CBELSA/TAPS [128, 129] shows that the real part of the $\eta^{\prime}$-nucleus optical potential is larger than its imaginary part giving a hope for the observation of the $\eta^{\prime}$ mesic nucleus.

The search for the $\eta$ and $\eta^{\prime}$ mesic nucleus is exciting and there are plans to continue these investigations in the future.

Acknowledgments We acknowledge support by the Polish National Science Center through grants No. 0320/B/H03/2011/40, 2011/01/B/ST2/00431, 2011/03/B/ST2/01847, by the FFE grants of the Research Center Jülich, by the Foundation for Polish Science (MPD programme), by the EU Integrated Infrastructure Initiative HadronPhysics Project under contract number RII3CT-2004-506078, and by the European Commission under the 7th Framework Programme through the Research Infrastructures action of the Capacities Programme, Call: FP7-INFRASTRUCTURES-2008-1, Grant Agreement N. 227431.

Open Access This article is distributed under the terms of the Creative Commons Attribution License which permits any use, distribution, and reproduction in any medium, provided the original author(s) and the source are credited.

\section{References}

1. Krusche, B., et al.: Photoproduction of mesons of nuclei: The photonuclear programs at ELSA and MAMI. J. Phys. Conf. Ser. 349, 012003 (2012)

2. Moskal, P., et al.: Close-to-threshold meson production in hadronic interactions. Prog. Part. Nucl. Phys. 49, 1 (2002)

3. Moskal P.: Close-to-threshold meson production in hadronic interactions. e-Print hep-ph/0408162

4. Wilkin, C.: Eta meson production in nucleon-nucleon collisions. Acta Phys. Polon. B 41, 2191-2200 (2010)

5. Fäldt, G., Johansson, T., Wilkin, C.: Near threshold production of $\eta$ and $\eta^{\prime}$ mesons in $\mathrm{p} p$ and $\mathrm{p} \mathrm{d}$ collisions. Phys. Scr. T 99, 146 (2002)

6. Pevsner, A., et al.: Evidence for a three pion resonance near 550 MeV. Phys. Rev. Lett. 7, 421 (1961)

7. Kalbfleisch, G.R., et al.: Observation of a nonstrange meson of mass 959 MeV. Phys. Rev. Lett. 12, 527 (1964)

8. Goldberg, M., et al.: Existence of a new meson of mass $960 \mathrm{MeV}$. Phys. Rev. Lett. 12, 546 (1964)

9. Bass, S.D.: Gluons and the eta-prime nucleon coupling constant. Phys. Lett. B 463, 286-292 (1999)

10. Batinić, M., et al.: Near threshold eta production in proton proton collisions. Phys. Scr. 56, 321 (1997)

11. Hanhart, C., Nakayama, K.: On the treatment of $\mathrm{N} \mathrm{N}$ interaction effects in meson production in $\mathrm{N} N$ collisions. Phys. Lett. B 454, 176 (1999)

12. Moskal, P., et al.: S wave eta-prime proton FSI: Phenomenological analysis of near threshold production of pi0, eta, and eta-prime mesons in proton proton collisions. Phys. Lett. B 482, 356-362 (2000)

13. Moskal, P., et al.: Experimental study of pp eta dynamics in the $p p \rightarrow p p \eta$ reaction. Phys. Rev. C 69, 025203 (2004)

14. Klaja, P., et al.: Measurement of the invariant mass distributions for the $p p \rightarrow p p \eta^{\prime}$ reaction at excess energy of $\mathrm{Q}=16.4 \mathrm{MeV}$. Phys. Lett. B 684, 11-16 (2010)

15. Meyer, H.O., et al.: Complete set of polarization observables in $p p \rightarrow p p \pi^{0}$ close to threshold. Phys. Rev. C 63, 064002 (2001)

16. Meyer, H.O., et al.: Measurement of partial wave contributions in $p p \rightarrow p p \pi^{0}$. Phys. Rev. Lett. 83, 5439 (1999)

17. Czyzykiewicz, R., et al.: Mechanism of the close-to-threshold production of the eta meson. Phys. Rev. Lett. 98, 122003 (2007)

18. Winter, P., et al.: First close to threshold measurement of the analyzing power $\mathrm{A}(\mathrm{y})$ in the reaction $p p \rightarrow p p \eta$. Eur. Phys. J. A 18, 355-357 (2003)

19. Winter, P., et al.: Analyzing power A(y) in the reaction $\mathbf{p} p \rightarrow p p \eta$ close to threshold. Phys. Lett. B544, 251-258 (2002); Erratum-ibid. B553, 339 (2003) 
20. Balestra, F., et al.: Exclusive eta production in proton-proton reactions. Phys. Rev. C 69, 064003 (2004)

21. Moskal, P., Hodana, M.: Study of the eta meson production with the polarised proton beam. J. Phys. Conf. Ser. 295, $012080(2011)$

22. Hodana, M., et al.: Studies of systematic uncertainties of polarization estimation for experiments with the WASA detector at COSY. Acta Phys. Polon. Supp. 6(4), 1041-1052 (2013)

23. Brauksiepe, S., et al.: COSY-11, an internal experimental facility for threshold measurements. Nucl. Instrum. Meth. A 376, 397-410 (1996)

24. Klaja, P., et al.: COSY-11: An experimental facillity for studying meson production in free and quasi-free nucleon-nucleon collisions. AIP Conf. Proc. 796, 160-163 (2005); hep-ex/0507055

25. Barsov, S., et al.: ANKE, a new facility for medium energy hadron physics at COSY-Jlich. Nucl. Instrum. \& Meth. A 462, 364 (2001)

26. Czerwinski, E., et al.: Determination of the total width of the $\eta^{\prime}$ meson. Phys. Rev. Lett. 105, 122001 (2010)

27. Amsler, C., et al.: Review of particle physics. Phys. Lett. B 667, 1 (2008)

28. Goslawski, P., et al.: New determination of the mass of the eta meson at COSY-ANKE. Phys. Rev. D 85, 112011 (2012)

29. Goslawski, P., et al.: High precision beam momentum determination in a synchrotron using a spin resonance method. Phys. Rev. Spec. Top. Accel. Beams 13, 022803 (2010)

30. Moskal, P., et al.: Upper limit for the cross-section of the overlapping scalar resonances $f(0)(980)$ and $a(0)(980)$ produced in proton proton collisions in the range of the reaction threshold. J. Phys. G 29, 2235-2246 (2003)

31. Bergdolt, A.M., et al.: Total cross-section of the $p p \rightarrow p p \eta$ reaction near threshold. Phys. Rev. D 48, 2969-2973 (1993)

32. Chiavassa, E., et al.: Measurement of the $p p \rightarrow p p \eta$ total cross-section between $1.265 \mathrm{GeV}$ and $1.5 \mathrm{GeV}$. Phys. Lett. B 322, 270-274 (1994)

33. Calen, H., et al.: The $p p \rightarrow p p \eta$ reaction near the kinematical threshold. Phys. Lett. B 366, 39-43 (1996)

34. Hibou, F., et al.: Comparison of eta and eta-prime production in the $p p \rightarrow p p \eta\left(\eta^{\prime}\right)$ reactions near threshold. Phys. Lett. B 438, 41-46 (1998)

35. Smyrski, J., et al.: Near threshold eta meson production in proton proton collisions. Phys. Lett. B 474, 182-187 (2000)

36. Abdel-Bary, M., et al.: Measurement of the eta production in proton proton collisions with the COSY time-of-flight spectrometer. Eur. Phys. J. A 16, 127-137 (2003)

37. Moskal, P., et al.: Invariant mass distributions for the $p p \rightarrow p p \eta$ reaction at $\mathrm{Q}=10 \mathrm{MeV}$. Eur. Phys. J. A 43, 131-136 (2010)

38. Petren, H., et al.: eta-meson production in proton-proton collisions at excess energies of 40 and $72 \mathrm{MeV}$. Phys. Rev. C 82, $055206(2010)$

39. Calen, H., et al.: Measurement of the quasifree $p n \rightarrow d \eta$ reaction near threshold. Phys. Rev. Lett. 79, 2642-2645 (1997)

40. Calen, H., et al.: Measurement of the quasifree $p n \rightarrow p n \eta$ reaction near threshold. Phys. Rev. C 58, 2667-2670 (1998)

41. Moskal, P., et al.: Near threshold production of the eta meson via the quasi-free $p n \rightarrow p n \eta$ reaction. Phys. Rev. C 79, 015208 (2009)

42. Kaptari, L.P., Kampfer, B., Semikh, S.S.: Tagging the $p n \rightarrow d \phi$ reaction by backward protons in $p d \rightarrow d \phi p(s p)$ processes. J. Phys. G 30, 1115 (2004)

43. Moskal, P., et al.: A method to disentangle single- and multi-meson production in missing mass spectra from quasi-free $p n \rightarrow p n X$ reactions. J. Phys. G 32, 629-641 (2006)

44. Abdel-Bary, M., et al.: Study of spectator tagging in the reaction $n p \rightarrow p p \pi$ with a deuteron beam. Eur. Phys. J. A 29, 353 (2006)

45. Abdel-Bary, M., et al.: Single $\pi^{-}$production in np collisions for excess energies up to $90 \mathrm{MeV}$. Eur. Phys. J. A 36, 7 (2008)

46. Duncan, F., et al.: Differential cross-section of the $p n \rightarrow p p((1) S(0)) \pi^{-}$reaction extracted from $p d \rightarrow p p p \pi^{-}$. Phys. Rev. Lett. 80, 4390 (1998)

47. Moskal, P., et al.: Eta-prime production in proton proton scattering close to threshold. Phys. Rev. Lett. 80, 3202-3205 (1998)

48. Moskal, P., et al.: Energy dependence of the near threshold total cross-section for the $p p \rightarrow p p \eta^{\prime}$ reaction. Phys. Lett. B 474, 416-422 (2000)

49. Balestra, F., et al.: Production of eta-prime mesons in the $p p \rightarrow p p \eta^{\prime}$ reaction at $3.67 \mathrm{GeV} / \mathrm{c}$. Phys. Lett. B 491, 29$35(2000)$

50. Khoukaz, A., et al.: Total and differential cross-sections for the $p p \rightarrow p p \eta^{\prime}$ reaction near threshold. Eur. Phys. J. A 20, 345350 (2004)

51. Czerwinski, E., et al.: Study of the N N eta-prime production with COSY-11. Acta Phys. Polon. B 45, 739 (2014). Acta Phys. Polon. B 45, 739 (2014). arXiv:1401.5924

52. Baru, V., et al.: On production of eta-prime mesons in p p collisions close to threshold. Eur. Phys. J. A 6, 445-450 (1999)

53. Sigg, D., et al.: The strong interaction shift and width of the ground state of pionic hydrogen. Nucl. Phys. A 600, 445 (1996)

54. Green, A.M., et al.: The eta-nucleon scattering length and effective range. Phys. Rev. C 55, 2167-2170 (1997)

55. Kaiser, N., Waas, T., Weise, W.: SU(3) chiral dynamics with coupled channels: Eta and kaon photoproduction. Nucl. Phys. A 612, 297 (1997)

56. Green, A.M., Wycech, S.: Uncertainties in the eta nucleon scattering length and effective range. e-Print Archive: nuclth/0009053

57. Green, A.M., Wycech, S.: A Coupled K matrix description of the reactions $\pi N \rightarrow \pi N, \pi N \rightarrow \eta N, \gamma N \rightarrow \pi N$ and $\gamma N \rightarrow \eta N$. Phys. Rev. C 60, 035208 (1999)

58. Bass, S.D., Thomas, A.W.: eta-eta mixing in eta-mesic nuclei. Acta Phys. Polon. B 41, 2239-2248 (2010)

59. Bass, S.D., Thomas, A.W.: QCD symmetries in eta and etaprime mesic nuclei. Acta Phys. Polon. B 45, 627 (2014) e-Print:arXiv: 1311.7248

60. Wolke, M.: Schwellennahe assoziierte Strangeness-Erzeugung in der Reaktion Acta Phys. Polon. B 45, $627(2014) p p \rightarrow$ $p p K^{+} K^{-}$am Experiment COSY-11. IKP Jül-3532 (1997)

61. Balestra, F., et al.: $K^{-}$meson production in the proton proton reaction at 3.67 GeV/c. Phys. Lett. B 468, 7-12 (1999)

62. Quentmeier, C., et al.: Near threshold $K^{+} K^{-}$meson-pair production in proton proton collisions. Phys. Lett. B 515, 276$282(2001)$ 
63. Winter, P., et al.: Kaon pair production close to threshold. Phys. Lett. B 635, 23-29 (2006)

64. Maeda, Y., et al.: Kaon pair production in proton proton collisions. Phys. Rev. C 77, 015204 (2008)

65. Silarski, M., et al.: Generalized Dalitz plot analysis of the near threshold $p p \rightarrow p p K^{+} K^{-}$reaction in view of the $K^{+} K^{-}$ final state interaction. Phys. Rev. C 3, 045202 (2009)

66. Ye, Q.J., et al.: The production of $K^{+} K^{-}$pairs in proton-proton collisions at $2.83 \mathrm{GeV}$. Phys. Rev. C 85, 035211 (2012)

67. Ye, Q.J., et al.: The production of $K^{+} K^{-}$pairs in proton proton collisions below the $\phi$ meson threshold. Phys. Rev. C 87, 065203 (2013)

68. Dzyuba, A., et al.: Coupled-channel effects in the $p p \rightarrow p p K^{+} K^{-}$reaction. Phys. Lett. B 668, 315-324 (2008)

69. Silarski, M., Moskal, P.: Combined analysis of the $K^{+} K^{-}$interaction using near threshold $p p \rightarrow p p K^{+} K^{-}$data. Phys. Rev. C 88, 025205 (2013)

70. Nakayama, K., et al.: An analysis of the reaction $p p \rightarrow p p \eta$ near threshold. Phys. Rev. C 68, 045201 (2003)

71. Deloff, A.: Phenomenology of $p p \rightarrow p p \eta$ reaction close to threshold. Phys. Rev. C 69, 035206 (2004)

72. Ceci, S., Švarc, A., Zauner, B.: Influence of the eta exchange to the eta production in proton-proton scattering. Acta Phys. Polon. Suppl. 2, 157-162 (2009)

73. Bass, S.D., Thomas, A.W.: Eta bound states in nuclei: A Probe of flavor-singlet dynamics. Phys. Lett. B 634, 368-373 (2006)

74. Bass, S.D.: Gluonic effects in eta and eta-prime physics. Phys. Scr. T 99, 96-103 (2002)

75. Klaja, J., et al.: Upper limit of the total cross section for the $p n \rightarrow p n \eta^{\prime}$ reaction. Phys. Rev. C 81, 035209 (2010)

76. Kaptari, L.P., Kampfer, B.: Eta-prime meson production in nucleon-nucleon collisions near the threshold. Eur. Phys. J. A 37, 69 (2008)

77. Berger, J., et al.: Identification of the $d p \rightarrow{ }^{3} \mathrm{He} \eta$ reaction very near threshold: Cross-section and deuteron tensor analyzing power. Phys. Rev. Lett. 61, 919-922 (1988)

78. Mayer, B., et al.: The reactions $p d \rightarrow^{3} \mathrm{He \eta}$ and $p d \rightarrow^{3} \mathrm{He} \pi^{+} \pi^{-}$near the eta threshold. Phys. Rev. C 53, 20682074 (1996)

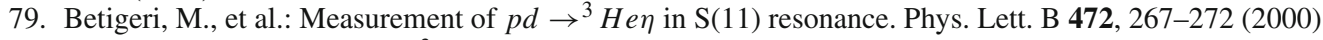

80. Adam, H.H., et al.: Hadronic ${ }^{3}$ He $\eta$ production near threshold. Phys. Rev. C 75, 014004 (2007)

81. Smyrski, J., et al.: Study of the ${ }^{3} \mathrm{He}-\eta$ system in d-p collisions at COSY-11. Acta Phys. Slov. 56, $213-219$ (2006)

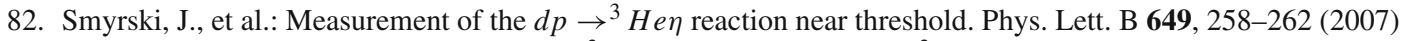

83. Mersmann, T., et al.: Precision study of the $\eta^{3} \mathrm{He}$ system using the $d p \rightarrow{ }^{3} \mathrm{He} \eta$ reaction. Phys. Rev. Lett. 98, 242301 (2007)

84. Frascaria, R., et al.: Total $d d \rightarrow \alpha \eta$ cross-sections near threshold. Phys. Rev. C 50, 537-540 (1994)

85. Willis, N., et al.: Eta-helium quasibound states. Phys. Lett. B 406, 14-19 (1997)

86. Wronska, A., et al.: Near threshold eta meson production in the $d d \rightarrow{ }^{4}$ He $\eta$ reaction. Eur. Phys. J. A 26, 421-428 (2005)

87. Budzanowski, A., et al.: Cross section and tensor analysing power of the $d d \rightarrow \eta \alpha$ reaction near threshold. Nucl. Phys. A 821, 193-209 (2009)

88. Hibou, F., et al.: Near threshold eta production in the $p d \rightarrow p d \eta$ reaction. Eur. Phys. J. A 7, 537 (2000)

89. Bilger, R., et al.: Measurement of the $p d \rightarrow p d \eta$ cross-section in complete kinematics. Phys. Rev. C 69, 014003 (2004)

90. Piskor-Ignatowicz, C., et al.: Near threshold eta meson production in dp collisions. Int. J. Mod. Phys. A 22, 528-532 (2007)

91. Krusche, B.: Photoproduction of $\eta$-mesons and $\eta \pi$ pairs off light nuclei. Acta Phys. Polon. B 45, 639 (2014)

92. Pfeiffer, M., et al.: Photoproduction of eta mesic ${ }^{3} \mathrm{He}$. Phys. Rev. Lett. 92, 252001 (2004)

93. Pheron, F., et al.: Coherent photoproduction of eta-mesons off ${ }^{3} \mathrm{He}$ - search for eta-mesic nuclei. Phys. Lett. B 709, 21 (2012)

94. Wilkin, C.: Light $\eta$-mesic Nuclei. Acta Phys. Polon. B 45, 603 (2014)

95. Khoukaz, A., et al.: Investigation of the ${ }^{3} \mathrm{He \eta}$ system with polarised beams at ANKE. Acta Phys. Polon. B 45, 655 (2014)

96. Wilkin, C.: Is there an eta ${ }^{3} \mathrm{He}$ quasi-bound state?. Phys. Lett. B 654, 92 (2007)

97. Haider, Q., Liu, L.C., et al.: Formation of an eta mesic nucleus. Phys. Lett. B 172, 257 (1986)

98. Bhalerao, R.S., Liu, L.C.: Off-shell model for threshold pionic eta production on a nucleon and for eta $\mathrm{N}$ scattering. Phys. Rev. Lett. 54, 865 (1985)

99. Green, A.M., Wycech, S.: Eta-nucleon scattering length and effective range uncertainties. Phys. Rev. C 71, 014001 (2005)

100. Wycech, S., Green, A.M., Niskanen, J.A.: Are there eta helium bound states?. Phys. Rev. C 52, 544 (1995)

101. Wilkin, C.: Near threshold production of eta mesons. Phys. Rev. C 47, 938-940 (1993)

102. Green, A.M., et al.: Eta-deuteron scattering. Phys. Rev. C 54, 1970 (1996)

103. Chrien, R.E., et al.: Search for bound states of the $\eta$ meson in light nuclei. Phys. Rev. Lett. 60, 2595 (1988)

104. Budzanowski, A., et al.: Search for eta-mesic nuclei in recoil-free transfer reaction. Phys. Rev. C 79, 012201 (2009)

105. Moskal, P., Smyrski, J.: Search for the $\eta$-mesic helium by means of COSY-11, WASA-at-COSY and COSY-TOF detector systems. Acta Phys. Pol. B 41, 2281 (2010)

106. Adlarson, P., et al.: Search for the eta-mesic ${ }^{4} \mathrm{He}$ with WASA-at-COSY detector. Phys. Rev. C 87, 035204 (2013)

107. Afanasiev, S.V., et al.: Search for eta-mesic nuclei in the reaction d + C at JINR. Phys. Part. Nucl. Lett. 8, 1073-1077 (2011)

108. Baskov, V.A., et al.: Studies of eta-mesic nuclei at the LPI electron synchrotron PoS Baldin-ISHEPP-XXI (2012) 102

109. Wycech, S., Krzemien, W.: Studies of mesic nuclei via decay reactions. Acta Phys. Polon. B 45, 745 (2014). e-Print: arXiv: 1401.0747

110. Skurzok, M., Moskal, P., Krzemien, W.: Search for He-eta bound states with the WASA-at-COSY facility. Prog. Part. Nucl. Phys. 67, 445 (2012)

111. Fujioka, H.: Search for eta-mesic nuclei at J-PARC. Acta Phys. Polon. B 41, 2261-2270 (2010)

112. Afanasiev, A.V. et al.: New status of the project " $\eta$-nuclei" at the NUCLETRON. Nucl. Phys. Proc. Suppl. 245, 173$176(2013)$

113. Wycech, S.: K-mesic nuclei versus eta-mesic nuclei. Acta Phys. Polon. B 41, 2201-2210 (2010)

114. Friedman, E., Gal, A., Mares, J.: $\eta$ nuclear bound states revisited. Phys. Lett. B 725, 334-338 (2013)

115. Cieply, A. et al.: In-medium eta-nucleon interactions and eta nuclear bound states. arXiv:1312.1547

116. Kelkar, N.G., et al.: Interaction of eta mesons with nuclei. Rep. Prog. Phys. 76, 066301 (2013) 
117. Hirenzaki, S., et al.: Formation of eta-mesic nuclei. Acta Phys. Polon. B 41, 2211-2220 (2010)

118. Jido, D., et al.: Medium effects to N(1535) resonance and eta mesic nuclei. Phys. Rev. C 66, 045202 (2002)

119. Inoue, T., Oset, E.: Eta in the nuclear medium within a chiral unitary approach. Nucl. Phys. A 710, 354 (2002)

120. Hirenzaki, S., Nagahiro, H.: Meson properties at finite density from mesic atoms and mesic nuclei: $\eta^{\prime}$ - and $\eta$-mesic nuclei. Acta Phys. Polon. B 45, 619 (2014)

121. Nagahiro, H., et al.: Formation of eta-prime(958)-mesic nuclei by (p,d) reaction. Phys. Rev. C 87, 045201 (2013)

122. Nagahiro, H., et al.: Eta-prime nucleus optical potential and possible eta-prime bound states. Phys. Lett. B 709, 87-92 (2012)

123. Nagahiro, H., Jido, D., Hirenzaki, S.: Formation of eta-mesic nuclei by (pi,N) reaction and $\mathrm{N}^{*}(1535)$ in medium. Phys. Rev. C 80, 025205 (2009)

124. Metag, V., et al.: Approved ELSA/03-20120BGO-OD

125. Tanaka, Y.K., et al.: Spectroscopy of eta mesic nuclei with (p,d) reaction. Few Body Syst. 54, 1263-1266 (2013)

126. Itahashi, K., et al.: Feasibility study of observing $\eta^{\prime}$ mesic nuclei with (p,d) reaction. Prog. Theor. Phys. 128, 601 (2012)

127. Nagahiro, H., Hirenzaki, S.: Formation of eta-prime(958)—mesic nuclei and axial U(A)(1) anomaly at finite density. Phys. Rev. Lett. 94, 232503 (2005)

128. Nanova, M., et al.: Transparency ratio in $\gamma A \rightarrow \eta^{\prime} A^{\prime}$ and the in-medium $\eta^{\prime}$ width. Phys. Lett. B 710, 600 (2012)

129. Nanova, M., et al.: Determination of the $\eta$ '-nucleus optical potential. Phys. Lett. B 727, 417-423 (2013) 